\title{
What has driven the evolution of multiple cone classes in visual systems: object contrast enhancement or light flicker elimination?
}

\author{
Shai Sabbah ${ }^{1 *}$ and Craig W Hawryshyn ${ }^{1,2}$
}

\begin{abstract}
Background: Two competing theories have been advanced to explain the evolution of multiple cone classes in vertebrate eyes. These two theories have important, but different, implications for our understanding of the design and tuning of vertebrate visual systems. The 'contrast theory' proposes that multiple cone classes evolved in shallow-water fish to maximize the visual contrast of objects against diverse backgrounds. The competing 'flicker theory' states that multiple cone classes evolved to eliminate the light flicker inherent in shallow-water environments through antagonistic neural interactions, thereby enhancing object detection. However, the selective pressures that have driven the evolution of multiple cone classes remain largely obscure.
\end{abstract}

Results: We show that two critical assumptions of the flicker theory are violated. We found that the amplitude and temporal frequency of flicker vary over the visible spectrum, precluding its cancellation by simple antagonistic interactions between the output signals of cones. Moreover, we found that the temporal frequency of flicker matches the frequency where sensitivity is maximal in a wide range of fish taxa, suggesting that the flicker may actually enhance the detection of objects. Finally, using modeling of the chromatic contrast between fish pattern and background under flickering illumination, we found that the spectral sensitivity of cones in a cichlid focal species is optimally tuned to maximize the visual contrast between fish pattern and background, instead of to produce a flicker-free visual signal.

Conclusions: The violation of its two critical assumptions substantially undermines support for the flicker theory as originally formulated. While this alone does not support the contrast theory, comparison of the contrast and flicker theories revealed that the visual system of our focal species was tuned as predicted by the contrast theory rather than by the flicker theory (or by some combination of the two). Thus, these findings challenge key assumptions of the flicker theory, leaving the contrast theory as the most parsimonious and tenable account of the evolution of multiple cone classes.

Keywords: Contrast hypothesis, Cone photoreceptors, Critical fusion frequency, Temporal contrast sensitivity, Opponent mechanisms, Color vision, Retina, Fish

\section{Background}

Multiple spectral classes of cones are found in the visual system of many vertebrates [1]. Comparison of the outputs of different cone classes enables color vision. Multiple cone classes appeared very early in vertebrate evolution, at least 540 MYA (million years ago) and perhaps as early as 700 MYA, prior to the separation of the jawed (Gnathostomata) and jawless (Agnatha) vertebrate lineages (approximately 485 MYA) [2,3]. This is based on the

\footnotetext{
* Correspondence: shai_sabbah@brown.edu

'Department of Biology, Queen's University, Kingston, Ontario K7L 3N6, Canada

Full list of author information is available at the end of the article
}

presence of five classes of cone-like photoreceptors in the jawless Southern Hemisphere lamprey, Geotria australis [4-6], and three cone classes in the jawed cartilaginous fishes (Chondrichthyes) [7-9]. Additionally, cone opsins have been suggested to evolve prior to rod opsins [10], indicating that photopic (bright light) vision preceded scotopic (dim light) vision, and suggesting that these early vertebrates occupied brightly-lit shallow-water environments [11]. However, although the evolution of visual pigments has been studied extensively $[1,4,6,10,12-20]$, the selective pressures that have driven the evolution of multiple cone classes in the eyes of vertebrates remain largely obscure.

\section{Biomed Central}


Two competing theories have been advanced to explain the evolution of multiple cone classes; both assumed that vision in ancestral vertebrates utilized multiple cone photoreceptor classes, with color vision evolving only later as a byproduct. The 'contrast theory' of Munz and McFarland and McFarland and Munz [13,14] proposed that multiple cone classes evolved in shallow-water fish to maximize the visual contrast between objects and their background. Indeed, a single visual pigment (either rod or cone) may suffice to maximize the visual contrast between a given object and background. However, the need to maximize contrast between diverse objects and backgrounds of varying brightness and spectral characteristics was suggested to favor the appearance of multiple cone classes. The competing 'flicker theory' presented by Maximov [21] proposed that multiple cone classes have evolved to allow elimination of the flicker (fluctuation in light intensity) produced by variation in the refraction of sunlight at the water surface [22-25]. It was argued that subtraction of the output of one cone class from another through antagonistic (opponent) neural interactions would filter out the light flicker, yielding a flicker-free representation of the visual scene and enhancing object detection. The flicker theory has received relatively little attention; however, it has remained a competitor of the contrast theory, leaving the forces that have driven the evolution of multiple cone classes an open question.

Both the contrast and flicker theories assume the presence of at least two cone classes that differ in spectral tuning. The flicker theory rests on three additional assumptions, one of which is the presence of antagonistic interactions between the output signals of the available cone classes. This assumption receives support from the presence of color-opponent horizontal cells [26,27] and the concentrically-antagonistic center-surround organization in retinal bipolar [28,29] and ganglion cells [30] in lower vertebrates. At least some of these color opponent mechanisms were probably present in early vertebrates that are represented today by the jawless lampreys [31-33]. However, two other critical assumptions of the flicker theory have so far not been seriously examined. First, it is assumed that 'the [light] fluctuations are colorless, that is, the intensity of light changes synchronously in different parts of the spectrum' [21]. Consequently, despite the strong fluctuations in light over the entire spectrum, the ratio of light intensities in two different parts of a spectrum would remain constant, and would depend only on the spectral properties of the viewed object. Second, the flicker theory assumes that 'the significant flicker of illumination inherent in the shallow-water environment complicated the visual process in the achromatic case, in particular preventing early detection of enemies' [21]. Thus, because light flicker would impair object detection, selection would favor removal of light flicker from the processed visual signal. These critical assumptions of the flicker theory have never been tested.

In this report, we evaluated the relative merits of the contrast and flicker theories. We first focused on the two largely untested assumptions of the flicker theory, and then asked whether the predictions of the two theories regarding the spectral tuning of cone pigments are supported by the evidence. We found that the amplitude and temporal frequency of light flicker are wavelength dependent and that the flicker may actually enhance the detection of objects, thus violating critical assumptions of the flicker theory. While this alone does not support the contrast theory, comparison of the contrast and flicker theories by means of chromatic contrast modeling under flickering illumination revealed that the spectral tuning of cone pigments of a focal cichlid species produced a large chromatic contrast between background and the body pattern of fish, and did not allow elimination of temporal fluctuations in the visual signal. This suggests that the visual system of the focal species is tuned as predicted by the contrast theory rather than by the flicker theory (or by some combination of the two).

\section{Results and discussion}

\section{Amplitude and temporal frequency of flicker are wavelength dependent}

The first critical assumption of the flicker theory is that 'the [light] fluctuations are colorless' [21], that is, the amplitude of the flicker and the distribution of its power across temporal frequencies are similar across the light spectrum. Only in this special case would the simple subtraction of outputs of different cone classes through color opponent channels produce a flicker-free visual signal, as posited in the original theory. To study the characteristics of underwater light flicker, we measured at high temporal resolution the downward and sideward irradiance from 310 to $750 \mathrm{~nm}$ at a range of water depths. Figure 1A,B illustrates light flicker time series of downward irradiance at $1 \mathrm{~m}$ depth and light wavelengths of 400 and $600 \mathrm{~nm}$. To estimate the amplitude of the flicker, we calculated the coefficient of variation (CV) of the time series of downward irradiance at each light wavelength. CV decreased with increasing water depth. However, consistent with past studies [22,34,35], CV increased monotonically at all depths toward longer light wavelengths, with long wavelengths 2.5 to 3 times more variable than short wavelengths (Figure $1 \mathrm{C}$ ). To study the temporal frequency of the light flicker, we calculated the power spectrum of the downward irradiance time series at each light wavelength. The power distribution of the flicker across frequencies varied with light wavelength (Figure 1D; Additional file 1A-D), consistent with a past study [34]. Additionally, the frequency distribution of flicker varied with water depth, and the dominant 


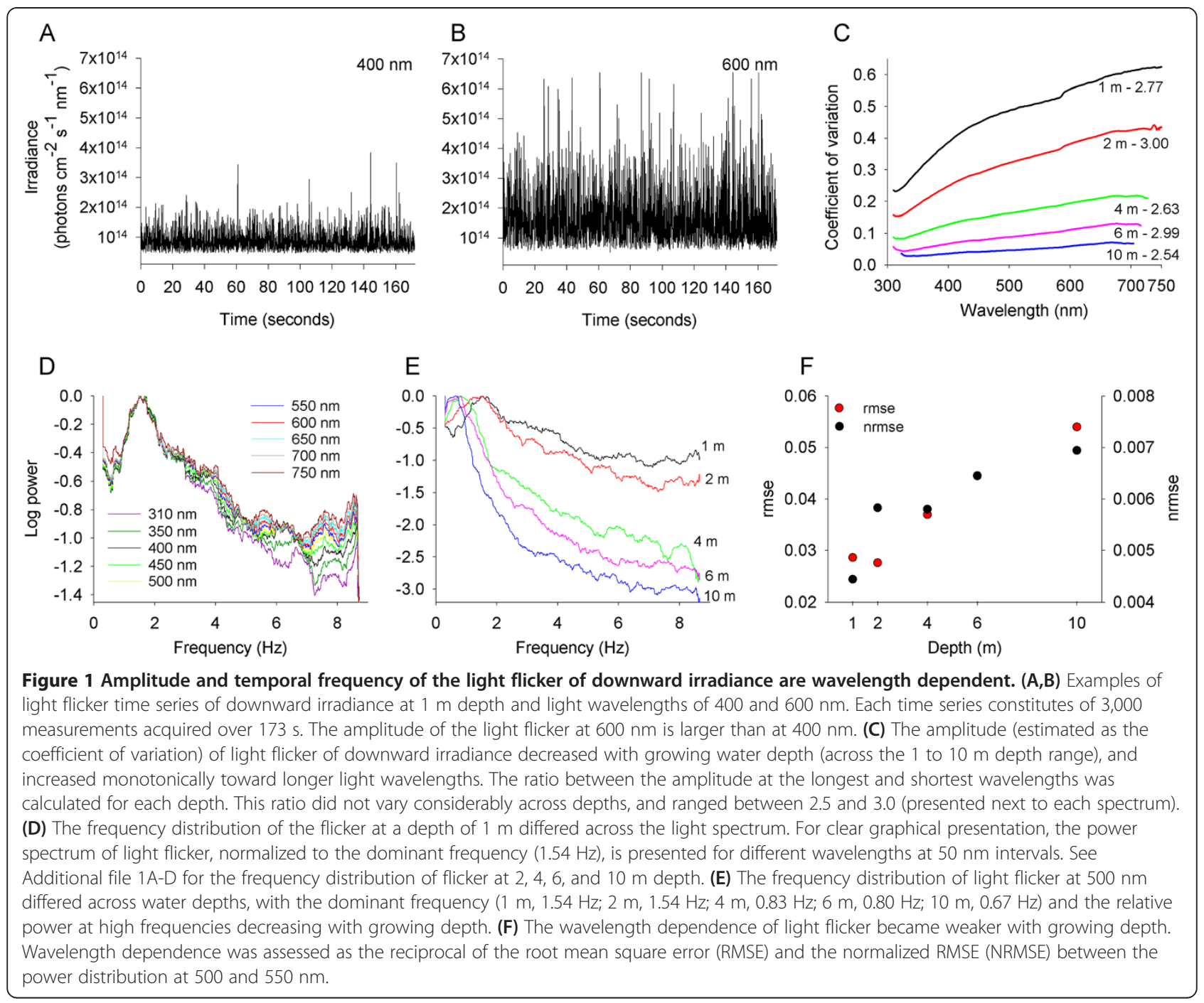

frequency of flicker decreased with increasing depth (Figure $1 \mathrm{E}$ ). The wavelength dependence of the power distribution of flicker also varied with water depth, such that the wavelength dependence of the power distribution became weaker with increasing depth (Figure 1F). These findings are consistent with variation observed in the wavelength dependence of the power distribution of light flicker across different viewing orientations [34]. (See Additional file 2 for similar analysis for sideward irradiance.) Light flicker is produced by the focusing and defocusing of sunlight rays refracted at the water surface $[22,24]$. The wavelength dependence of the amplitude and temporal frequency of the flicker arises largely from variation in the scattering of light across the spectrum. Scattering at short wavelengths by molecules and small particles in the atmosphere and water is generally more pronounced than at long wavelengths. This causes short-wavelength light to be more diffused and consequently less affected by the wave-focusing phenomenon than long-wavelength light. Therefore, our results demonstrate that the amplitude of the flicker and the distribution of its power across temporal frequencies vary across the light spectrum, violating the flicker theory's first assumption.

\section{Subtraction of cone outputs through opponent channels does not produce a flicker-free visual signal}

We examined the effect of the wavelength dependence of light flicker on the output of cones and on the capacity of a simple subtraction of cone outputs to eliminate the flicker from the processed signal. To this end, we calculated the cone output (estimated by the quantum catch of cone pigments) when viewing an achromatic target (reflectance $=50 \%$ across the spectrum) under flickering illumination in a focal species, Metriaclima zebra (a Lake Malawi cichlid). The cone pigment complement found in adult $M$. zebra includes the SWS1 $(368 \mathrm{~nm}), \mathrm{Rh} 2 \mathrm{~b}(484 \mathrm{~nm})$, and 
Rh2a $(523 \mathrm{~nm})$ pigments (wavelength values represent the wavelength of maximum absorbance of cones, $\lambda_{\max }$ ) [36]. The target viewed was assumed to be illuminated by flickering sideward irradiance at a depth of $1 \mathrm{~m}$, and cones were assumed to be adapted to the mean sideward irradiance at the same depth. (See the Methods section for detailed description of how cone and opponent channel outputs were calculated).

Cone output varied considerably over time under flickering illumination, with the variation in cone output (estimated as the standard deviation over time) decreasing when moving from SWS1 (0.070), through Rh2b (0.026), and to Rh2a (0.013) (cone output is unitless because it is normalized by the adapting light) (Figure 2A). The output among the three cones varied because long wavelength pigments such as Rh2b and Rh2a show broad sensitivity functions, with both the $\alpha$ - and $\beta$-absorption bands included in the 300 to $800 \mathrm{~nm}$ spectrum that might be used for vision. By sampling the light flicker across the spectrum, the broad sensitivity functions act to reduce the variation of cone output produced under wavelengthdependent flickering illumination. In contrast, short wavelength pigments such as SWS1 show narrow sensitivity functions, with only the $\alpha$-absorption band included in the 300 to $800 \mathrm{~nm}$ spectrum. These narrow sensitivity functions act to increase the variation in cone output.

The configuration of color opponent channels in $M$. zebra is currently unknown. Thus, we modeled three possible opponent channels, that is, SWS1-Rh2b, SWS1Rh2a, and Rh2b-Rh2a. Output of opponent channels subjected to flickering illumination varied over time, showing variation largely comparable to that in the

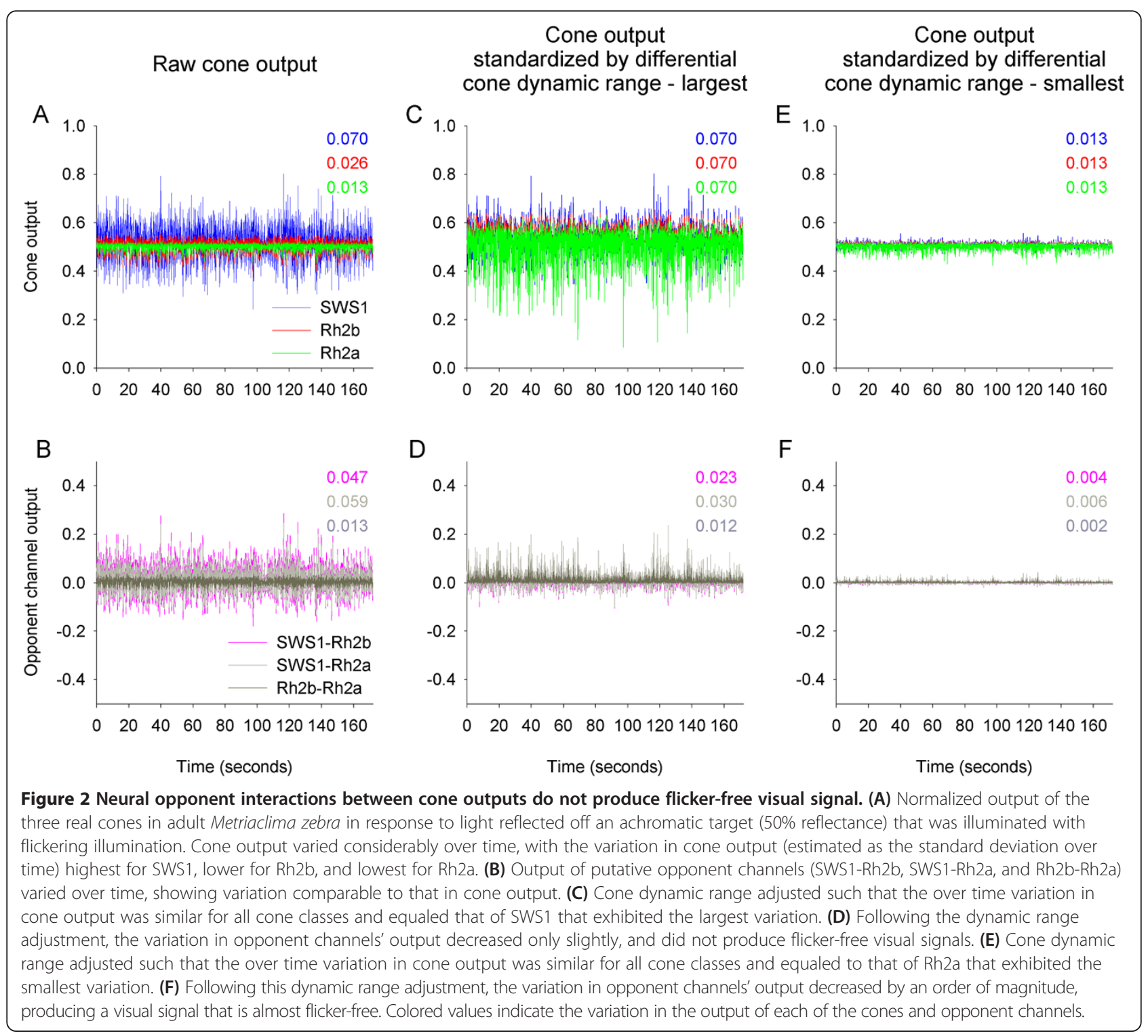


output of cones (SWS1-Rh2b, 0.047; SWS1-Rh2a, 0.059; and $\mathrm{Rh} 2 \mathrm{~b}-\mathrm{Rh} 2 \mathrm{a}, 0.013$ ) (Figure 2B). Therefore, contrary to the prediction of the flicker theory, simple subtraction of the output of one cone class from that of another through opponent interactions would not produce flicker-free output signal.

Certain modifications to the flicker theory would potentially allow the intended generation of flicker-free visual signals. An obvious way to generate a flicker-free visual signal would be to route the cone output through a low pass filter. This would attenuate any high frequency components in the signal, possibly producing flicker-free opponent channel signals. Such filtration, however, would inevitably reduce the temporal resolution by which the animal is sampling the environment, compromising the animal's ability to detecting fast moving and changing stimuli, and reacting to them. Furthermore, considering the wavelength dependence of the power distribution of flicker (discussed above), even the low frequency components that are allowed to pass the filter would differ slightly between different cone classes; that is, even opponent channels that only use the low frequency components would not produce a flicker-free signal. Therefore, the use of low pass filtration for generating flicker-free visual signals comes with high cost, and is unlikely to provide a selective advantage.

Adjusting the dynamic range of cones would also potentially allow generation of flicker-free opponent channel output. For example, if the dynamic range of cones were to vary such that variation in cone output across the three cone classes was equalized over time, then subtraction of cone output through opponent channels would potentially produce a flicker-free signal. To test this possibility, we artificially adjusted the dynamic range of cones so that the variation over time in cone output was similar for all cone classes and equaled that of the SWS1 cone that exhibited the largest variation (Figure $2 \mathrm{C}$ ). Following the dynamic range adjustment, the variation in opponent channels' output decreased only slightly, and did not produce flicker-free visual signals (SWS1-Rh2b, 0.023; SWS1-Rh2a, 0.030; and Rh2b-Rh2a, 0.012) (Figure 2D). Additionally, such differential adjustment of the dynamic range of cones would reduce the resolution in which the incident color signals (radiance) that vary in intensity are being sampled, potentially compromising the discrimination between targets of relatively close spectral reflectance characteristics. We also artificially adjusted the dynamic range of cones such that the variation in cone output over time was similar for all cone classes, but now, equaled to that of the $\mathrm{Rh} 2 \mathrm{a}$ cone that exhibited the smallest variation (Figure 2E). Following this second dynamic range adjustment, the variation in opponent channels' output decreased by an order of magnitude, producing a visual signal that is almost flicker-free (SWS1-Rh2b, 0.004; SWS1-Rh2a, 0.006; and Rh2b-Rh2a, 0.002) (Figure 2F). Thus, such a mechanism may theoretically generate a flicker-free output of opponent channels. Note however, that such differential adjustment of the dynamic range of cones to span the dynamic range of the cone that shows the narrowest dynamic range would result in failure to sample many incident color signals that fall outside of this narrow dynamic range. This would substantially compromise the detection of targets of various spectral reflectance characteristics, which are illuminated by a range of irradiance levels. Therefore, this possibility is also highly unlikely to have a selective advantage.

Nevertheless, the implementation of complex antagonistic interactions between cone classes (for example, SWS1 + Rh2b - Rh2a), may potentially allow the intended generation of flicker-free visual signals. However, as our light flicker measurements show, the dominant frequency, the frequency distribution of flicker, and the wavelength dependence of the power distribution of flicker all vary with water depth. This suggests that a fixed, 'wired' compensation for the wavelength dependence of flicker would fail when an animal moves in the water column or views objects at different lines of sight; behaviors that are common to many fish. In summary, contrary to the prediction of the flicker theory, simple subtraction of the output of one cone class from that of another through opponent interactions would not produce a flicker-free output signal. Moreover, neither fixed low pass filtration nor adjustment of the dynamic range of cones would likely to be favored. Thus, although there might be a mechanism by which flicker-free visual signals would be generated under flickering illumination, the likelihood of such a possibility is low, and the likelihood that such a possibility would be favored either by natural or sexual selection is even lower.

\section{Temporal frequency of light flicker matches the frequency where maximum contrast sensitivity in fish is attained}

A second assumption of the flicker theory is that flicker interferes with object detection. However, by generating periodic changes in the retinal image, flicker may enhance perception [37] and detection of coarse (low spatial frequency) patterns [38]. The flicker is analogous to the flashing of an artificial light, such as a turn signal on a car; it is visually prominent because of the extreme brightness change, and possibly because of an unknown visual alertness system. This enhancement of object appearance would be most efficient if the temporal frequency of the flicker matched the frequency where maximum contrast sensitivity $\left(\mathrm{F}_{\max }\right)$ is attained $[35,39,40]$. Unfortunately, to date, the complete temporal contrast sensitivity function has been determined for only one fish 
species, the goldfish (Carassius auratus) [41], while the temporal contrast sensitivity of fish has often been studied through the measurement of the critical fusion frequency (CFF), the frequency at which temporally modulated light stimuli appear to fuse and have constant brightness. The temporal contrast sensitivity function varies with the adaptation state of the eye, the mean intensity of the modulated light stimulus, the visual angle subtended by the stimulus beam, and the temperature. Nevertheless, it still takes on a rather simple and easily modeled function [42-44], where $\mathrm{F}_{\max }$ typically ranges between $10 \%$ and $20 \%$ of CFF [41,45-48]. Thus, to accommodate comparison between the frequency of wave-induced light flicker and the $F_{\max }$ of various fish species, as a first-order approximation, $\mathrm{F}_{\max }$ was estimated as $15 \%$ of CFF, and then as either $10 \%$ or $20 \%$ of CFF, covering the possible realistic range of relationships between $\mathrm{F}_{\max }$ and CFF.

We compiled CFF data for 47 fish species, representing 41 genera, 34 families, and 14 orders, including both bony and cartilaginous fishes. See Additional file 3 for compilation of CFF data and $F_{\max }$ estimates with reference to the light adaptation regime and temperature used during experiments, as well as the habitat and depth distribution of each species. $F_{\max }$ varied with stimulus intensity, light adaptation regime, and temperature. Nonetheless, with $\mathrm{F}_{\max }$ estimated as $15 \%$ of CFF, the distribution of $\mathrm{F}_{\max }$ values across frequency corresponded well to the power spectrum of the light flicker, being highest at the dominant frequency of the flicker (Figure 3A). To support our findings further, we calculated the cumulative power of the flicker across temporal frequencies, which, unlike the dominant frequency of the flicker, is rather robust to variation in surface wave conditions. The median $F_{\max }$ for dim stimuli, $1.8 \mathrm{~Hz}$, matched the frequency below which close to $50 \%$ of the cumulative power of flicker was found at $1 \mathrm{~m}$ depth. The median $\mathrm{F}_{\max }$ for bright stimuli, $3.5 \mathrm{~Hz}$, matched the frequency below which between $50 \%$ and $90 \%$ of the cumulative power of flicker was found at $1 \mathrm{~m}$ depth (Figure 3B). The relative power at high frequencies decreased with increasing depth. Yet, the median $F_{\max }$ for both dim and bright stimuli matched the frequency below which $99 \%$ of the cumulative power was found at $10 \mathrm{~m}$ depth, for which the relative power at high frequencies was the lowest (Figure 3B; Additional file 1E-G). A similar trend was observed when $F_{\max }$ was estimated as either $10 \%$ or $20 \%$ of CFF (Additional file 4). Therefore, these findings suggest that light flicker may enhance the detection by fish of underwater objects under a range of light intensities and water depths, violating the flicker theory's second assumption.

Note that the correspondence between the $F_{\max }$ of fish and the frequency of flicker might potentially break if the fish species included in the analysis were to change. This is especially important if $F_{\max }$ varied across environmental categories such as habitat type and depth. Species were classified based on their habitat type as being either 'benthic', 'benthopelagic', or 'pelagic' species. Species were also classified as inhabiting either 'deep' (typically found in depths $>30 \mathrm{~m}$ ), 'shallow' (typically found in depths $<30 \mathrm{~m}$ ), or both deep and shallow ('shallow-deep') habitats. As for the comparison between $F_{\max }$ and flicker frequency, the analysis included all 35 fish species for which $F_{\max }$ data were available for both dim/natural and bright light stimuli ( $75 \%$ of cases) and were obtained under dark adaptation $(80 \%$ of eligible pairs). The percentage of species inhabiting shallow (40\%) and shallow-deep (46\%) habitats was similar, whereas the percentage of strictly deep-water species was lower (14\%). Moreover, the percentage of benthopelagic species $(63 \%)$ was higher than those of either benthic (17\%) or pelagic (20\%) species (Figure 3C). Thus, if $F_{\max }$ differs between habitat or depth categories, this may bias the comparison between $F_{\max }$ and flicker frequency. To test this possibility, we examined the effect of water depth and habitat type on the $F_{\max }$ of fish. $F_{\max }$ for bright stimuli differed significantly across habitat types (randomization test (RT), df $=2, N=35, P=0.0098$ ) (Figure 3D). Post-hoc analysis revealed that $F_{\max }$ in benthic species was significantly lower than in either benthopelagic $(P=0.0095)$ or pelagic $(P=0.0012)$ species; $\mathrm{F}_{\max }$ in benthopelagic and pelagic species did not differ significantly $(P=0.272)$. Moreover, $\mathrm{F}_{\max }$ for bright stimuli did not differ significantly across depth categories (RT, $\mathrm{df}=2, N=35, P=0.4472$ ) (Figure $3 \mathrm{E}$ ). $\mathrm{F}_{\max }$ did not differ significantly across depth categories also when fish from the 'shallow-deep' depth category were pooled with those from the 'shallow' category (RT, $\mathrm{df}=1, N=35$, $P=0.6676)$ or with the 'deep' category (RT, $\mathrm{df}=1, N=35$, $P=0.3231$ ). Therefore, $F_{\max }$ of fish does not vary with habitat depth; however, $\mathrm{F}_{\max }$ of benthic species is lower than in the other habitats. Consequently, if benthic species, that represent only $17 \%$ of the species analyzed, were to be represented better in the analysis, this would shift the distribution (and median) of $F_{\max }$ values toward lower frequencies. Interestingly, inspection of Figure 3A,B reveals that this would actually improve the correspondence between $F_{\max }$ of fish and the frequency of flicker, further supporting our conclusions regarding a correspondence between the $F_{\max }$ of fish and flicker frequency.

\section{Contrast theory versus flicker theory: a comparative analysis}

The violation of its two critical assumptions substantially undermines support for the flicker theory as originally formulated. However, this alone does not support the contrast theory. Interestingly, considering the wavelength dependence of the light flicker, the two competing theories would predict opposite evolutionary pathways. Using 

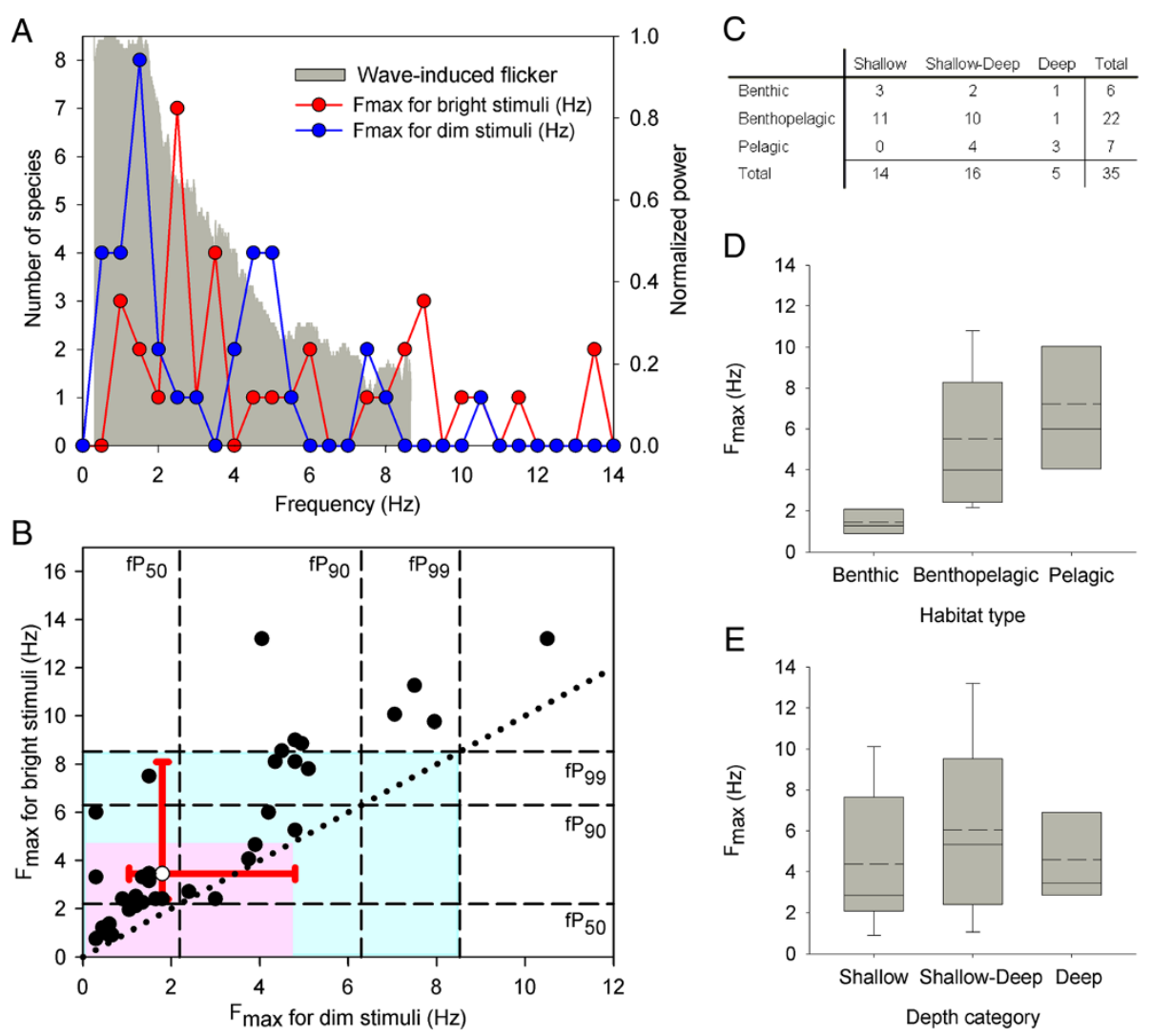

Figure 3 Light flicker can enhance the detection of underwater objects. (A) Comparison between the frequency of light flicker in downward irradiance and the $F_{\max }$ of fish for dim and bright stimuli. The distribution of $F_{\max }$ values across frequency corresponded well to the power spectrum of flicker across depths. Depicted power spectrum (gray shaded area) represents the envelope of flicker power across the $1 \mathrm{~m}$ and $10 \mathrm{~m}$ depth range. (B) Comparison between the cumulative power of the flicker and $\mathrm{F}_{\max }$ for dim and bright stimuli (closed circles). The indices $\mathrm{fP}_{50}, \mathrm{fP}_{90}$, and $\mathrm{fP}_{99}$ stand for the frequencies that correspond to 50,90, and 99 percent of the cumulative power of flicker across frequencies, averaged across the light spectrum. Vertical and horizontal dashed lines represent the $\mathrm{fP}_{50}, \mathrm{fP}_{90}$, and $\mathrm{fP}_{99}$ values at $1 \mathrm{~m}$ depth. Gray and pink shaded areas represent the frequency range enclosed by the $99 \%$ cumulative power bounds at $1 \mathrm{~m}$ and $10 \mathrm{~m}$ depth, respectively. (See Additional file $1 \mathrm{E}-\mathrm{G}$ for the cumulative power indices as a function of light wavelength.) $F_{\max }$ data points typically fell above the identity (dotted) line, indicating larger $F_{\max }$ values for bright stimuli. The median $F_{\max }$ equaled 1.8 and $3.5 \mathrm{~Hz}$, for dim and bright stimuli, respectively (open circle; bidirectional red error bars represent the 25th and 75th percentiles). (C) Summary of the number of species inhabiting the various habitats and depths examined. (D,E) Association between the $F_{\max }$ of fish for bright stimuli and the habitat type (D) and water depth (E). Box specifications: mean (dashed), median (solid), 25th and 75th percentiles; whiskers: 10th and 90th percentiles. For consistency, all analyses included only fish species for which $\mathrm{F}_{\max }$ data were available for both dim/natural and bright light stimuli (75\% of cases), and were obtained under dark adaptation (80\% of eligible pairs; $n=35$ ); different $F_{\max }$ reports for a given species were averaged.

multiple cone classes to increase the contrast of objects would have favored cone classes whose peak sensitivities are far apart. By contrast, using multiple cone classes to eliminate the light flicker would have favored cone classes whose peak sensitivities are the closest possible to each other. Nonetheless, providing conclusive evidence for the dominance of a given evolutionary pathway would require comparing the specific spectral locations of cone pigments of every fish species to the spectrum of ambient light under various conditions and for various behavioral contexts. Such a task is clearly impossible. Here instead, we chose to use a case study, a Lake Malawi cichlid, to assess whether the spectral location of cone pigments has been shaped as predicted by the contrast theory or by the flicker theory, or by both.

For each opponent channel, we calculated responses to a range of naturally occurring body color patterns of fish (measured as diffused spectral reflectance) and the backgrounds against which the fish might be viewed under flickering illumination. The difference between these responses $(\Delta C)$ is a measure of the chromatic contrast of the particular opponent channel under these specific conditions. The model assigned spectral sensitivities to the cones based on the known characteristics of the cone pigments in this fish. The cichlid Metriaclima zebra belongs to the rock-dwelling Mbuna clade. In these 
species, males defend a territory in between rocks and perform elaborate displays to approaching females against the background of a vertical rock. Thus, following the contrast theory, the visual system would be designed to ensure large $\Delta C$ magnitude between the body patterns of males and the background of rocks. However, following the flicker theory, the visual system would be structured to ensure small $\Delta C$ variation under flickering illumination. The prediction regarding the contrast theory follows from the notion that the spectral location of visual pigments has evolved to maximize the chromatic contrast between the color pattern of males and their background. However, at least another possibility exists. That is, that the spectral location of visual pigments in females has been shaped by natural selection, and that males evolved body color patterns to maximize the chromatic contrast against their background, under those pre-existing visual system characteristics of females ('sensory bias' hypothesis) [49]. The specific selection forces that have driven the spectral diversification of visual pigments in Lake Malawi cichlids are currently largely unknown [50].

$\Delta C$ was calculated for two putative types of opponent channels: (i) a channel that compares the outputs of one single cone and one double cone, and (ii) a channel that compares the outputs of two double cones. Lake Malawi cichlids typically display three cone pigments (but see [36,51]), with short-wavelength sensitive pigments (typically $\lambda_{\max }<456 \mathrm{~nm}$; SWS1, SWS2b, and SWS2a) occupying single cones, and longer-wavelength sensitive pigments (typically $456<\lambda_{\max }<560 \mathrm{~nm}$; Rh2b, Rh2a, and LWS) occupying double cones [18,52-54]. Accordingly, $\lambda_{\max }$ of modeled pigments in single cones ranged between 365 and $455 \mathrm{~nm}$ (every $5 \mathrm{~nm}$ ), whereas $\lambda_{\max }$ of modeled pigments in double cones ranged between 460 and $560 \mathrm{~nm}$ (every 5 $\mathrm{nm})$. For each modeled pigment pair, $\Delta C$ was calculated for every single combination of fish body pattern $(n=87)$ and rock background $(n=8)$. Thereafter, we determined the pigment pairs that produced the largest $\Delta C$ magnitude ('max $\Delta C$ mag'; estimated as the time average $\Delta C$ ) and the smallest $\Delta C$ variation (' $\min \Delta C$ var'; estimated as the standard deviation in $\Delta C$ over time) over 100 consecutive high-temporal resolution sideward irradiance measurements (total duration $=6 \mathrm{~s}$ ). The larger the $\Delta C$ magnitude, the larger are the chances that the chromatic contrast between object and background would exceed threshold (as soon as $\Delta C$ exceeds the threshold, objects can be detected with nearly $100 \%$ probability). The smaller the $\Delta C$ variation, the more efficient is the elimination of flicker from the output of opponent channels. This analysis was repeated for the two most extreme water depths examined in this study, $1 \mathrm{~m}$ and $10 \mathrm{~m}$. (See Methods for detailed description of the modeling procedure and Additional file 5 for data used for modeling.)
The variation and magnitude of $\Delta C$ for an opponent channel formed by one single cone and one double cone located at a depth of $1 \mathrm{~m}$ are plotted for various cone pigment pairs in Figure 4A and B, respectively. Calculated values are displayed in the white tags on the isovalue contours across the various pigment combinations. Values rise from blue to red regions on the plots. Figure 4C,D presents findings for the same model channel located at a depth of $10 \mathrm{~m}$. Results for similar analysis of an opponent pair of double cones are plotted in Figure 4E-H. For the opponent channel formed by comparison of one single cone and one double cone, the max $\Delta C$ mag pigment pair constituted of pigments with $\lambda_{\max }$ that were far apart (365 and $560 \mathrm{~nm}$ for $1 \mathrm{~m}$ depth; 410 and 560 for $10 \mathrm{~m}$ depth; Figure 4B,D, green X symbols), while the $\min \Delta C$ var pigment pair constituted of pigments with $\lambda_{\max }$ that were closer together (455 and 460 for both 1 and $10 \mathrm{~m}$ depth; Figure 4A,C, red X symbols). Similarly, for the opponent channel formed by comparison of two double cones, the max $\Delta C$ mag pigment pair constituted of pigments with $\lambda_{\max }$ that were far apart (460 and $560 \mathrm{~nm}$ for both 1 and $10 \mathrm{~m}$ depth), while the $\min \Delta C$ var pigment pairs constituted of two identical pigments with $\lambda_{\max }$ spanning the realistic range for double cones (460 to $560 \mathrm{~nm}$ ), for both 1 and $10 \mathrm{~m}$ depth (Figure 4E-H). Thus, the $\lambda_{\max }$ of the real pigments in M. zebra (368, 484, and $523 \mathrm{~nm}$; black X symbols), and the spectral separation between the pigments, resemble those of the max $\Delta C$ mag pigments more than they resemble those of the min $\Delta$ Cvar pigments.

Next, we examined quantitatively how the $\Delta C$ magnitude produced by the actual cone pigments in $M$. zebra corresponds to the $\Delta C$ magnitudes produced by the $\max \Delta C$ mag and $\min \Delta C$ var pigments. Because of the large sample size $(n=696$ for each of the groups, 87 body patterns $\times 8$ rock substrates), slight differences between treatment groups most often resulted in statistically significant differences between groups $(P<0.05)$. We therefore chose to report observed effect size (that is, $\eta^{2}$ values) to allow evaluation of the magnitude of difference between groups. The larger the effect size, the larger is the difference between treatment groups. Unless specified differently, $P$ for all comparisons was $<0.05$.

For the opponent channel formed by one single cone and one double cone (Figure 4I,J), $\Delta C$ magnitude differed between the real (black), $\min \Delta C$ var (red), and $\max \Delta C$ mag (green) pigment pairs (RT, $\mathrm{df}=3, N=696$ for each group, $\eta^{2}=0.243$ and 0.186 for 1 and $10 \mathrm{~m}$ depth). Post-hoc analysis revealed that $\Delta C$ magnitude for the $\min \Delta C$ var pigment pair was smaller than for either the real pigment pairs (SWS1-Rh2b, df $=1, \eta^{2}=0.312$ and 0.253 for 1 and $10 \mathrm{~m}$ depth; SWS1-Rh2a, $\mathrm{df}=1, \eta^{2}=0.362$ and 0.289 for 1 and $10 \mathrm{~m}$ depth) or the $\max \Delta C$ mag pigment pair ( $\mathrm{df}=1, \eta^{2}=0.422$ and 0.446 for 1 and $10 \mathrm{~m}$ depth). In contrast, $\Delta C$ magnitude for the real and $\max \Delta C$ mag 


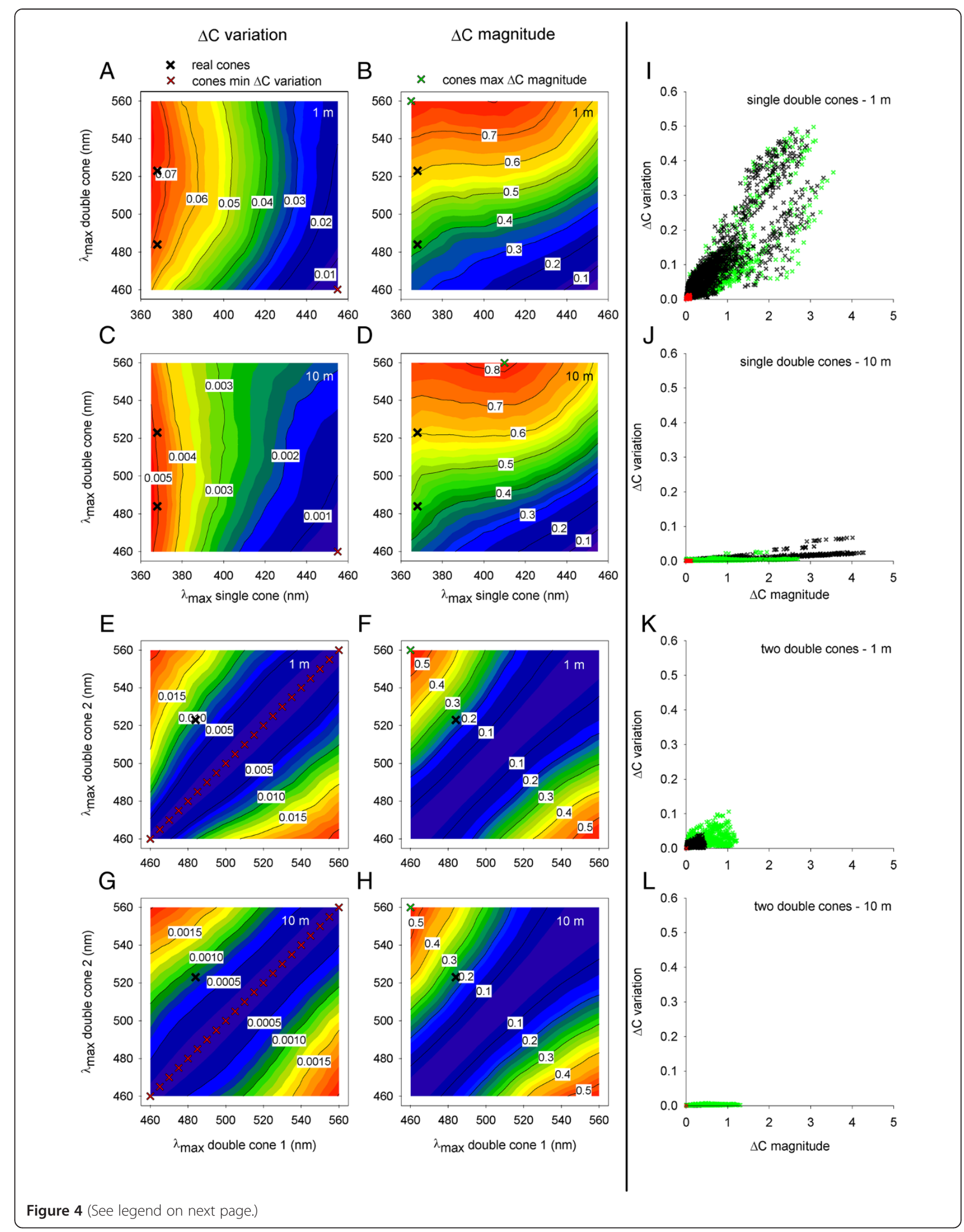


(See figure on previous page.)

Figure 4 Spectral location of pigments in Metriaclima zebra is tuned to allow large chromatic contrast between fish pattern and background. (A-H) Median $\Delta C$ between fish pattern and background. $\Delta C$ was modeled for opponent channels that compare the outputs of one single cone and one double cone (A-D), and channels that compare the outputs of two double cones (E-H). The various colors and tags in the contour plots represent different median $\Delta C$ values. The max $\Delta C$ mag pigment pairs comprised pigments with $\lambda_{\max }$ that were far apart, and resembled the $\lambda_{\max }$ of the real pigments in $M$. zebra $(368,484$, and $523 \mathrm{~nm})$. In contrast, the min $\Delta$ Cvar pigment pairs consisted of pigments with $\lambda_{\max }$ that were closer together. This trend held for both opponent channel types and for both $1 \mathrm{~m}$ and $10 \mathrm{~m}$ depth. (I-L) Scatterplots illustrating the relationship between $\Delta C$ magnitude and $\Delta C$ variation for the real (black), $\min \Delta C$ var (red), and max $\Delta C$ mag (green) pigment pairs. $\Delta C$ magnitude produced by the real pigment pair resembled the $\Delta C$ amplitude produced by the max $\Delta C$ mag pigment pair better than it resembled the $\Delta C$ amplitude produced by the min $\Delta C$ var. This was observed at both $1 \mathrm{~m}$ and $10 \mathrm{~m}$ depth, and for both opponent channel types (I,J and $\mathbf{K}, \mathbf{L}) . \Delta C$ variation at $10 \mathrm{~m}$ depth was smaller than at $1 \mathrm{~m}$ depth. Regardless of water depth, however, the real and max $\Delta C \mathrm{mag}$ pigment pairs produced variation in $\Delta C$ that was larger than in the min $\Delta C$ var pigment pairs. Sample size, $n=696$ for either the real, min $\Delta C$ var, or max $\Delta C$ mag pigment pairs, for each of the opponent channels and water depths examined.

pigment pairs differed only slightly (RT, $\mathrm{df}=2, \eta^{2}=0.027$ and 0.005 for 1 and $10 \mathrm{~m}$ depth), as did the $\Delta C$ magnitude for the real pigment pairs (RT, $\mathrm{df}=1, \eta^{2}=0.013$ and 0.006 for 1 and $10 \mathrm{~m}$ depth). Therefore, the $\Delta C$ magnitude produced by the real and $\max \Delta C$ mag pigment pairs is largely similar, but considerably larger than the $\Delta C$ magnitude produced by the min $\Delta$ Cvar pigment pair.

For the opponent channel formed by two double cones (Figure 4K,L), $\Delta C$ magnitude differed between the real, $\min \Delta C$ var, and max $\Delta C$ mag pigment pairs $(\mathrm{RT}, \mathrm{df}=2$, $N=696$ for each group, $\eta^{2}=0.555$ and 0.556 for 1 and $10 \mathrm{~m}$ depth). Post-hoc analysis revealed that $\Delta C$ magnitude for the $\min \Delta$ Cvar pigment pair was smaller than for either the real pigment pair $\left(\mathrm{Rh} 2 \mathrm{~b}-\mathrm{Rh} 2 \mathrm{a}, \mathrm{df}=1, \eta^{2}=0.555\right.$ and 0.556 for 1 and $10 \mathrm{~m}$ depth) or the max $\Delta C$ mag pigment pair ( $\mathrm{df}=1, \eta^{2}=0.585$ and 0.585 for 1 and $10 \mathrm{~m}$ depth). In contrast, $\Delta C$ magnitude for the real and $\max \Delta C$ mag pigment pairs differed less considerably $(\mathrm{RT}, \mathrm{df}=1$, $\eta^{2}=0.315$ and 0.316 for 1 and $10 \mathrm{~m}$ depth). Thus, the $\Delta C$ magnitude produced by the real pigment pair resembles the $\Delta C$ magnitude produced by the $\max \Delta C$ mag pigment pair better than it resembles the $\Delta C$ magnitude produced by the $\min \Delta C$ var. Therefore, at both 1 and $10 \mathrm{~m}$ depth, the real and $\max \Delta C$ mag pigment pairs are more effective than the min $\Delta C$ var pigment pair in producing large $\Delta C$ magnitude. These results suggest that the spectral location of pigments in M. zebra is almost optimally tuned to allow the largest possible $\Delta C$ magnitude between fish pattern and background, as predicted by the contrast theory.

Figure 4I-L allows comparison of the $\Delta C$ variation produced by the real cone pigments in $M$. zebra with that produced by the $\max \Delta C \operatorname{mag}$ and $\min \Delta C$ var pigments. For the opponent channel formed by one single cone and one double cone (Figure 4I,J), the flicker-induced variation in $\Delta C$ differed between the real, $\min \Delta C$ var, and max $\Delta C$ mag pigment pairs (RT, $\mathrm{df}=3, N=696$ for each group, $\eta^{2}=0.189$ and 0.197 for 1 and $10 \mathrm{~m}$ depth). Post-hoc analysis revealed that $\Delta C$ variation for the $\min \Delta C$ var pigment pair was smaller than for either the real pigment pairs (SWS1-Rh2b, df $=1, \eta^{2}=0.314$ and 0.269 for 1 and $10 \mathrm{~m}$ depth; SWS1-Rh2a, df $=1$, $\eta^{2}=0.319$ and 0.263 for 1 and $10 \mathrm{~m}$ depth) or the $\max \Delta C$ mag pigment pair $\left(\mathrm{df}=1, \eta^{2}=0.318\right.$ and 0.372 for 1 and $10 \mathrm{~m}$ depth). However, $\Delta C$ variation for the real and $\max \Delta C$ mag pigment pairs did not differ significantly at 1 $\mathrm{m}$ depth (RT, $\mathrm{df}=2, P=0.257, \eta^{2}=0.001$ ), but did differ at $10 \mathrm{~m}$ depth (RT, df $\left.=2, P<0.001, \eta^{2}=0.071\right)$; At both 1 and $10 \mathrm{~m}$ depth, $\Delta C$ variation for the real pigment pairs did not differ significantly $(\mathrm{RT}, \mathrm{df}=1, P=0.798$ and $\eta^{2}=0.0008$ for $1 \mathrm{~m}$ depth, $P=0.666$ and $\eta^{2}<0.0001$ for $10 \mathrm{~m}$ depth).

For the opponent channel formed by two double cones (Figure $4 \mathrm{~K}, \mathrm{~L}$ ), the flicker-induced variation in $\Delta C$ differed between the real, $\min \Delta C$ var, and $\max \Delta C$ mag pigment pairs (RT, $\mathrm{df}=2, N=696$ for each group, $\eta^{2}=0.458$ and 0.563 for 1 and $10 \mathrm{~m}$ depth). Post-hoc analysis revealed that $\Delta C$ variation for the $\min \Delta C$ var pigment pair was smaller than for either the real pigment pair (Rh2b-Rh2a, $\mathrm{df}=1, \eta^{2}=0.458$ and 0.563 for 1 and $10 \mathrm{~m}$ depth) or the $\max \Delta C$ mag pigment pair $\left(\mathrm{df}=1, \eta^{2}=0.488\right.$ and 0.598 for 1 and $10 \mathrm{~m}$ depth). In contrast, $\Delta C$ variation for the real and max $\Delta$ Cmag pigment pairs differed less substantially (RT, df $=1, \eta^{2}=0.023$ and 0.318 for 1 and $10 \mathrm{~m}$ depth). Therefore, the real pigment pairs are often slightly more effective than the max $\Delta C$ mag pigment pairs in eliminating the variation in $\Delta C$. Nevertheless, at both 1 and $10 \mathrm{~m}$ depth, the real and $\max \Delta C$ mag pigment pairs are considerably less effective than the $\min \Delta C$ var pigment pairs in eliminating the variation in $\Delta C$. These results suggest that the spectral location of pigments in $M$. zebra is poorly tuned to allow elimination of temporal fluctuations in the visual signal, in contrast to the prediction of the flicker theory.

Taken together, three lines of evidence suggest that the spectral location of cone pigments in M. zebra has been shaped as predicted by the contrast theory rather by the flicker theory. These are: (i) similarity in cone spectral locations between the $\max \Delta C$ mag pigments and the real pigments in $M$. zebra, (ii) greater efficiency of the real and max $\Delta C$ mag pigment pairs in producing large $\Delta C$ magnitude as compared to the $\min \Delta C$ var pigment pair, and (iii) lower efficiency of the real and $\max \Delta C$ mag 
pigment pairs in eliminating the variation in $\Delta C$, as compared to the min $\Delta C$ var pigment pair. Thus, the spectral location of pigments in M. zebra is tuned to produce large $\Delta C$ magnitude between fish pattern and background, and is poorly tuned to allow elimination of temporal fluctuations in the visual signal. That is, the visual system of $M$. zebra is tuned as predicted by the contrast theory rather than by the flicker theory (or by both theories).

\section{Conclusions}

Our results show that the amplitude of the light flicker and the distribution of its power across temporal frequencies vary across the light spectrum, violating the flicker theory's first assumption. We examined the effect of the wavelength dependence of light flicker on the output of cones and found that, contrary to the prediction of the flicker theory, simple subtraction of the output of one cone class from that of another through opponent interactions would not produce a flicker-free output signal. Moreover, neither fixed low pass filtration nor adjustment of dynamic range of cones would likely to be favored. Thus, although there might be a mechanism by which flicker-free visual signals would be generated under flickering illumination, the likelihood of such a possibility is low. Importantly, even if such generation of a flicker-free visual signal would prove possible, our results show that the temporal frequency of flicker matches the frequency where sensitivity is maximal in a wide range of fish species, suggesting that the flicker may potentially enhance the detection of objects. Thus, there appears to be no real need to eliminate the flicker, because, in contrast to the accepted belief and the second assumption of the flicker theory, the flicker can most likely improve the detection of objects rather than degrade it. The violation of its two critical assumptions suggests little support for the flicker theory as originally formulated. While this alone does not support the contrast theory, comparison of the contrast and flicker theories by means of chromatic contrast modeling under flickering illumination revealed that the visual system of our focal species was tuned as predicted by the contrast theory rather than by the flicker theory. This suggests that the main factor that has tuned the spectral locations of cone pigments is the optimization of visual contrast. Thus, we propose that the contrast theory, stating that multiple cone classes evolved to maximize the visual contrast between objects and backgrounds, is the most parsimonious at present. This result may have important implications for our understanding of the adaptive significance of the number and spectral tuning of cone pigments and the characteristics of retinal networks in vertebrate visual systems.

\section{Methods}

\section{Measurement of underwater light flicker}

The study was conducted on 21 July 2008 at a near-shore site at Cape Maclear, Nankumba Peninsula, Lake Malawi. The sampling site (14 01' 26.42” S 34 49' 25.91” E) was located on the southern shore of Thumbi West Island. This site is exposed to wind and wave action [55] and has a rock-sand transition depth of approximately $12 \mathrm{~m}$.

To study the light flicker characteristics, downward and sideward irradiance was measured at a high sampling rate. Irradiance was measured using a thermoelectrically cooled spectroradiometer (QE65000, Ocean Optics, Dunedin, FL, USA) connected to a $30 \mathrm{~m}$ optical fiber (ZPK600-30 Ultraviolet-visible, Ocean Optics) that was fitted with a cosine corrector (diameter $=3.9 \mathrm{~mm}$; CC-3-Ultraviolet, Ocean Optics). This diameter of the cosine corrector was expected to accurately capture the irradiance fluctuations at nearsurface depths. However, we cannot exclude the possibility of miscapturing fluctuations of small spatial scale, typically encountered at depths smaller than $1 \mathrm{~m}$ [22]. The spectroradiometer employed a 1,024 $\times$ 58-element square silicon charge-coupled device (CCD) array, configured with a $25 \mu \mathrm{m}$ slit and a variable blaze wavelength grating ( $\mathrm{HC}-1$, groove density $=300 \mathrm{~mm}^{-1}$, Ocean Optics $)$, resulting in an effective spectral resolution of $1.9 \mathrm{~nm}$ 'Full Width at Half Maximum' (FWHM) between 200 and 950 $\mathrm{nm}$. The spectroradiometer's integration time was set to $25 \mathrm{~ms}$ (theoretical sampling frequency $=40 \mathrm{~Hz}$ ) to allow for the highest possible sampling rate while ensuring sufficiently high signal-to-noise ratio. In practice, however, due to a time constant between successive readings, the actual sampling frequency was $17.34 \mathrm{~Hz}$. Thus, 3,000 measurements were saved over $173 \mathrm{~s}$, constituting a measurement time series. The spectroradiometer setup was calibrated for absolute irradiance prior to measurement using a calibrated halogen-deuterium dual light source (200 to 1,000 nm, DH-2000-CAL, Ocean Optics). The optical fiber head was mounted on a $1 \mathrm{~m}$ tall tripod, 1, 2, 4, 6, and $10 \mathrm{~m}$ below the water surface, and readings were saved on a laptop computer placed on a boat. To prevent shading, the boat was positioned as far as possible from the tripod and never between the tripod and the sun. Irradiance measurements were conducted under clear blue sky, at 12:20 to 14:09 (local time), with solar zenith angles of $46^{\circ}$ to $55^{\circ}$, and under light winds of $1.8 \mathrm{~m} / \mathrm{s}$.

Note that various elements included in the spectroradiometer setup may introduce spectrally-specific variation in the measurement. These include: (i) light attenuation in the fiber optic cable, (ii) light absorbance by the spectroradiometer's mirrors, (iii) reflectance efficiency of the spectroradiometer's grating, and (iv) response of the CCD detector. However, the wavelength dependence of these elements has been removed by calibrating the spectroradiometer setup (including the spectroradiometer, 
fiber optic and cosine corrector) for absolute irradiance. Therefore, spectrally-specific differences in the design of the spectroradiometer setup likely had little effect (if any) on the observed wavelength dependence of light flicker.

Although vision is essentially a task of low spectral resolution and high temporal resolution radiance detection, we have chosen to measure irradiance at a relatively low temporal resolution for several reasons. First, we aimed at investigating the wavelength dependence of light flicker, so we chose to sacrifice some temporal resolution while ensuring precise representation of irradiance across the spectrum. Second, we chose to focus on characterization of light flicker at temporal frequencies corresponding to the $F_{\max }$ of fish;, typically ranging between 2 and $4 \mathrm{~Hz}$ (Figure 3). Third, the power of light flicker typically declines steeply with increasing temporal frequency. For example, the power of light flicker at a frequency of 8.67 $\mathrm{Hz}$ (our frequency limit considering a sampling frequency of $17.34 \mathrm{~Hz}$ ) was reported to be approximately 5 to 200 fold smaller than that at the dominant frequency at depths of 0.86 to $2.84 \mathrm{~m}$ [56]. Indeed, to fully capture the highestfrequency irradiance fluctuations, it would be necessary to use a high rate (for example, $1 \mathrm{kHz}$ ) radiometric measurement system [22]. However, the relatively low frequency of $F_{\text {max }}$ in fish as well as the steep decline of light flicker's power with increasing frequency, suggest a limited effect of high-frequency irradiance fluctuations on the appearance of objects.

\section{Analysis of amplitude and temporal frequency of light flicker}

To standardize the 3,000 readings included in each irradiance time series, the noise level (measured with the tip of the cosine corrector blocked) was subtracted from each spectroradiometer reading, and the resulting reading in relative counts was converted into photon irradiance. Wavelengths at which irradiance was lower than $3 \times 10^{11}$ photons $\mathrm{cm}^{2} / \mathrm{s} / \mathrm{nm}$ were designated as unreliable and removed from further analysis. To estimate the amplitude of the light flicker, we calculated the $\mathrm{CV}$ of each irradiance time series. $\mathrm{CV}$ is commonly used in describing the variation in irradiance and radiance flicker [22,34,35,57]. To study the frequency characteristics of the wave-induced light flicker, we calculated the power spectrum of temporal frequencies for each irradiance time series at a light wavelength resolution of $1 \mathrm{~nm}$. Specifically, the discrete Fourier transform (DFT) was calculated for each time series by using the fast Fourier transform (FFT) algorithm, and while applying a Hamming frequency window that is appropriate for analyzing closely spaced sine waves [58]. Additionally, as indices of the distribution of power across frequencies, we calculated the $\mathrm{fP}_{50}, \mathrm{fP}_{90}$, $\mathrm{fP}_{99}$ that stand for the frequencies corresponding to 50, 90, and 99 percent of the cumulative power of the light flicker.
Although dependent on the frequency range examined, the $\mathrm{fP}$ indices describe the modulations experienced by an observer reasonably well [40]. Finally, to assess the wavelength dependence of the power distribution of light flicker, we calculated the root mean square error (RMSE) and the normalized RMSE (NRMSE) between the power distribution at 500 and $550 \mathrm{~nm}$. Irradiance at these wavelengths is highest and most reliable, and thus, the calculated RMSE and NRMSE are likely to serve as good estimates for wavelength dependence. NRMSE equaled RMSE divided by the difference between the maximum and minimum power across the spectrum.

\section{Modeling the magnitude and variation of chromatic contrast under flickering illumination}

Chromatic contrast modeling was performed following Kelber et al. [59] and Cummings [60]. The quantum catch of each cone photoreceptor, $Q_{i}$, when viewing a given color patch of the fish was calculated according to:

$$
Q_{\mathrm{i}}=\int_{300}^{800} R_{\mathrm{t}}(\lambda) E_{\mathrm{h}}(\lambda) A_{\mathrm{i}}(\lambda) T(\lambda) d \lambda
$$

where $R_{\mathrm{t}}(\lambda)$ is the spectral reflectance of the target (ranging 0 to 1 ), $E_{\mathrm{h}}(\lambda)$ is the normalized sideward spectral irradiance incident on the object (ranging 0 to 1 ), $A_{\mathrm{i}}$ $(\lambda)$ is the normalized absorbance of cone photoreceptor $i$ (ranging 0 to 1 ), and $T(\lambda)$ is the normalized spectral transmission of the ocular media (ranging 0 to 1 ). Similarly, the quantum catch of each photoreceptor when viewing the background of a rock substrate (the stimulus fish might be viewed against) was calculated using Equation 1 where $R_{\mathrm{t}}(\lambda)$ was substituted by the spectral reflectance of the substrate, $R_{\mathrm{b}}(\lambda)$. The absorbance of photoreceptors was estimated as the empirical absorbance templates of visual pigments given by Govardovskii et al. [61]. See below detailed procedures for the measurement of spectral reflectance of the body pattern of fish and rock substrate (approximated by diffuse reflectance [62]), and spectral transmission of the ocular media (approximated by the transmission of the lens [63]). The quantum catch of photoreceptors should ideally be estimated using absorbtance rather than absorbance spectra, with the former depending on the transverse specific density of pigments and the outer segment length of photoreceptors. However, transverse specific density and outer segment length data in M. zebra (our focal species) and in African cichlids as a whole is largely unexplored, with the few available reports providing incomplete and contradicting values [64-66]. Thus, photoreceptor quantum catch was estimated using absorbance spectra.

To account for the light adaptation properties of photoreceptors, photoreceptor quantum catches, $Q_{\mathrm{i}}$, were 
normalized to the adapting background irradiance by the von Kries coefficients, $K_{\mathrm{i}}$ :

$$
q_{\mathrm{i}}=K_{\mathrm{i}} Q_{\mathrm{i}}
$$

These $K_{\mathrm{i}}$ coefficients were chosen so that the quantum catches for the adapting irradiance is constant, that is:

$$
K_{\mathrm{i}}=1 / \int_{300}^{800} \bar{E}_{\mathrm{h}}(\lambda) A_{\mathrm{i}}(\lambda) T(\lambda) d \lambda
$$

where $\bar{E}_{\mathrm{h}}(\lambda)$ is the normalized mean sideward spectral irradiance that was assumed to adapt the fish eye, calculated as the time-average of 3,000 consecutive sideward irradiance measurements. We modeled two types of opponent channels: (i) a channel that compares the outputs of one single cone and one double cone $\left[C_{\mathrm{sd}}\right]$, and (ii) a channel that compares the outputs of two double cones $\left[C_{\mathrm{dd}}\right]$ :

$$
\begin{aligned}
& C_{\mathrm{sd}}=q_{\mathrm{s}}-q_{\mathrm{d}} \\
& C_{\mathrm{dd}}=q_{\mathrm{d} 1}-q_{\mathrm{d} 2}
\end{aligned}
$$

Thereafter, we calculated the chromatic contrast $(\Delta C)$, formed by comparison of the output of a given opponent channel when viewing the body color pattern of fish and the background against which it might be viewed:

$$
\Delta C=C_{\mathrm{t}}-C_{\mathrm{b}}
$$

where $C_{\mathrm{t}}$ and $C_{\mathrm{b}}$ represent the output of a given opponent channel when viewing the object and the background, respectively. $\Delta C$ amplitude was estimated as the time average of $\Delta C$ over 100 consecutive hightemporal resolution sideward irradiance measurements (total duration $=6 \mathrm{~s}$ ), and $\Delta C$ variation was estimated as the standard deviation in $\Delta C$ over time. The use of standard deviation, rather than coefficient of variation, to estimate the variation in $\Delta C$ is appropriate because the quantum catches of photoreceptors were already normalized to the mean adapting irradiance; this effectively rendered the quantum catches of the different cones to be of the same magnitude.

\section{Measurement of spectral reflectance of the body pattern of fish}

Diffuse spectral reflectance of the body pattern of $M$. zebra $(n=87)$ was measured at $1-\mathrm{nm}$ intervals using a spectroradiometer (effective spectral resolution $=2.06$ nm FWHM for 200 to 950 nm; USB2000, Ocean Optics) connected to one arm of a $2 \mathrm{~m}$ bifurcated optical fiber (BIF600-2 Ultraviolet-visible, Ocean Optics). The other arm of the fiber was connected to a high output light source (200 to 1,000 nm; DH-2000-BAL, Ocean Optics). The common end of the bifurcated fiber was fitted with a flat black reflectance probe that showed a $3 \mathrm{~mm}$ diameter tip, cut at an angle of $45^{\circ}$. A measurement of a Spectralon diffuse reflectance standard (WS-1-SL, Ocean Optics) was taken as $100 \%$ reflectance, and a dark measurement was taken as zero reflectance. Fish were immersed in $500 \mathrm{ml}$ water containing $2 \mathrm{ml}$ of 1:10 clove oil:ethanol solution immediately after capture until the fish reached stage III anesthesia [67]. Reflectance was measured at 16 to 23 different points across the submerged fish body of 5 individuals. All experimental and animal care procedures were approved by Queen's University Animal Care Committee under the auspices of the Canadian Council for Animal Care.

\section{Measurement of spectral reflectance of rock substrate}

Diffuse spectral reflectance of rock substrate $(n=8)$ was measured at a near-shore site in Lake Malawi $\left(14^{\circ} 00^{\prime}\right.$ 58.02" S $34^{\circ} 48^{\prime} 33.29^{\prime \prime}$ E) [68]. Rock reflectance was measured using a custom-built probe that included a diving flashlight (mini Q40, Underwater Kinetics, Poway, CA, USA) and a fiber-coupled spectroradiometer (Jaz, Ocean Optics). The tip of the flashlight was fitted with an adaptor that held the optical fiber (QP600-2 Ultraviolet-visible, Ocean Optics) oriented at an angle of $45^{\circ}$ to the examined surface. The far side of the adaptor included a ring of black foam that sealed the reflectance probe against the surface examined. A SCUBA diver held the reflectance probe against rock substrates while readings were acquired and saved on a laptop computer in a boat. The irradiance spectrum of the flashlight allowed reliable reflectance measurements between 370 and $800 \mathrm{~nm}$, and the spectroradiometer configuration resulted in an effective spectral resolution of $2.06 \mathrm{~nm}$ (FWHM) across this range. A measurement of a Spectralon diffuse reflectance standard was taken as $100 \%$ reflectance, and a dark measurement was taken as zero reflectance.

\section{Measurement of spectral transmission of fish lens}

Spectral transmission of the fish lens was measured following a protocol described elsewhere [69,70]. Lenses were surgically removed from the eyes and were mounted in a hole that was drilled in a black plastic block fitted inside a standard sample cuvette. Transmission measurements between 300 and $750 \mathrm{~nm}$ were carried out using a bench-top spectrophotometer (Cary 300; Varian, Palo Alto, CA, USA) and were normalized between 0 and 1 . For each fish $(n=3)$, six to ten transmission measurements were acquired from both lenses and averaged.

\section{Statistical analysis}

$F_{\max }$ of fish from different water depth categories and habitat types did not follow normal distribution (Kolmogorov-Smirnov test) and differed in variance (Leven's test). Thus, to test the effect of water depth and habitat type on $F_{\max }$, we used ANOVA permutation tests, with the difference between the means of the various depth 
categories and habitat types as a test statistic ( $\mathrm{R}$ package 'ImPerm', maximum number of iterations $=50,000, \alpha=$ 0.05) [71]. Similarly, chromatic contrast $(\Delta C)$ between fish pattern and background for the real, $\min \Delta C$ var, and $\max \Delta C$ mag pigment pairs did not follow normal distribution and their variance differed between groups. Thus, permutation tests were used also to test the effect of pigment pair on $\Delta C$ amplitude and variation. To allow evaluation of the magnitude of difference in $\Delta C$ amplitude and variation between pigment pair treatments, effect size was estimated as $\eta^{2}$ (= sum of squares treatment/sum of squares total). Statistical analysis was performed using R 3.0.0 (The R Foundation for Statistical Computing).

\section{Additional files}

Additional file 1: Temporal frequency of light flicker is wavelength dependent across various water depths. (A-D) The frequency distribution of the flicker in downward irradiance at a depth of $2 \mathrm{~m}$ (A), 4 $m(B), 6 m(C)$, and $10 m$ (D) differed across the light spectrum. For clear graphical presentation, the power spectrum of light flicker, normalized to the dominant frequency $(1.54 \mathrm{~Hz}$ for $2 \mathrm{~m}, 0.83 \mathrm{~Hz}$ for $4 \mathrm{~m}, 0.80 \mathrm{~Hz}$ for 6 $\mathrm{m}, 0.67 \mathrm{~Hz}$ for $10 \mathrm{~m}$ ) is presented for different wavelengths at $50 \mathrm{~nm}$ intervals. (E-G) Cumulative power of wave-induced flicker across wavelengths and water depths. As indices of the distribution of flicker power across temporal frequencies, we calculated the $\mathrm{fP}_{50}, \mathrm{fP}_{90}$, and $\mathrm{fP}_{99}$ that stand for the temporal frequencies that correspond to 50,90, and 99 percent of the cumulative power of wave-induced flicker. $\mathrm{fP}_{50}, \mathrm{fP}_{90}$, and $\mathrm{fP}_{99}$ increased toward longer light wavelengths, further supporting the wavelength dependence of the temporal frequency structure of flicker. Note that deeper in the water column, the irradiance at both ends of the spectrum was too low to be considered reliable (see Methods for criteria for excluding data points); therefore, the spectral range presented narrows with depth.

Additional file 2: Amplitude and temporal frequency of the light flicker in sideward irradiance are wavelength dependent. $(A, B)$

Examples of light flicker time series of sideward irradiance at $1 \mathrm{~m}$ depth and light wavelengths of 400 and $600 \mathrm{~nm}$. The amplitude of the light flicker at $600 \mathrm{~nm}$ is larger than at $400 \mathrm{~nm}$. (C) The amplitude of light flicker in sideward irradiance decreased with growing water depth, and increased monotonically toward longer light wavelengths. The ratio between the amplitude at the longest and shortest wavelengths did not vary considerably across depths, and ranged between 2.26 and 2.79 (presented next to each spectrum). (D) The frequency distribution of the flicker at a depth of $1 \mathrm{~m}$ differed across the light spectrum. The power spectrum of light flicker, normalized to the dominant frequency $(1.69 \mathrm{~Hz})$, is presented for different wavelengths at $50 \mathrm{~nm}$ intervals. The frequency distribution of flicker at 2, 4, 6, and $10 \mathrm{~m}$ depth also differed between wavelengths (not presented). (E) The frequency distribution of light flicker at $500 \mathrm{~nm}$ differed across water depths, with the dominant frequency $(1 \mathrm{~m}, 1.69 \mathrm{~Hz} ; 2 \mathrm{~m}, 1.30 \mathrm{~Hz} ; 4 \mathrm{~m}, 0.83 \mathrm{~Hz} ; 6 \mathrm{~m}, 0.78 \mathrm{~Hz} ; 10 \mathrm{~m}$, $0.59 \mathrm{~Hz}$ ) and the relative power at high frequencies decreasing with growing depth. (F) The wavelength dependence of light flicker became weaker with growing depth.

Additional file 3: Compilation of critical fusion frequency (CFF) and the frequency at which maximum contrast sensitivity is attained ( $F_{\text {max }}$, estimated as $15 \%$ of CFF) in fish. Frequencies are given in $\mathrm{Hz}$.

Additional file 4: Comparison between the frequency of light flicker in downward irradiance and two realistic estimates of $F_{\max }$. $F_{\text {max }}$ was estimated as either $10 \%(\mathbf{A}, \mathbf{B})$ or $20 \%(\mathbf{C}, \mathbf{D})$ of CFF. $(A, C)$ The distribution of $F_{\max }$ values across frequency corresponded well to the power spectrum of flicker across depths. Note, however, that estimation of $F_{\max }$ as $20 \%$ of CFF resulted in $F_{\text {max }}$ values that often exceeded the sampling frequency limit of light flicker. Depicted power spectrum (shaded gray) represents the envelope of flicker power across the $1 \mathrm{~m}$ and $10 \mathrm{~m}$ depth range. (B,D) Comparison between the cumulative power of the flicker and $\mathrm{F}_{\max }$ for dim and bright stimuli (closed circles).

Conventions for the indices of the distribution of power of flicker across frequencies $\left(\mathrm{fP}_{50}, \mathrm{fP}_{90}\right.$, and $\left.\mathrm{fP}_{99}\right)$, plot specifications, and species included in the analysis are the same as in Figure 3A,B. For estimation of $F_{\max }$ as $10 \%$ of CFF, the median $F_{\max }$ equaled 1.2 and $2.3 \mathrm{~Hz}$, for dim and bright stimuli, respectively (open circle; red error bars represent the 25th and 75th percentiles). The median $\mathrm{F}_{\max }$ for $\mathrm{dim}$ and bright stimuli matched the frequency below which approximately $50 \%$ of the cumulative power of flicker was found at $1 \mathrm{~m}$ depth. For estimation of $\mathrm{F}_{\max }$ as $20 \%$ of CFF, the median $F_{\text {max }}$ equaled 2.4 and $4.6 \mathrm{~Hz}$, for dim and bright stimuli, respectively. The median $F_{\max }$ for $\operatorname{dim}$ and bright stimuli matched the frequency below which between $50 \%$ and $90 \%$ of the cumulative power of flicker was found at $1 \mathrm{~m}$ depth. For both $\mathrm{F}_{\max }$ estimates, the median $F_{\text {max }}$ for dim and bright stimuli matched the frequency below which $99 \%$ of the cumulative power at $10 \mathrm{~m}$ depth was found.

\section{Additional file 5: Spectra used for chromatic contrast modelling.}

$(\mathbf{A}, \mathbf{B})$ A total of 100 spectra of sideward irradiance at a water depth of 1 $m(A)$ and $10 m(B)$. These spectra were taken as the irradiance that illuminated the stimulus fish and the vertical rock substrate. (C,D) Mean spectral sideward irradiance at a depth of $1 \mathrm{~m}(\mathrm{C})$ and $10 \mathrm{~m}$ (D) that was taken as the irradiance that adapted the viewer fish eye. (E) Spectral reflectance of the body pattern of fish $(n=87)$. (F) Spectral reflectance of diverse rock substrates $(n=8)$. (G) Spectral transmission of the lens in Metriaclima zebra. (H) Spectral absorbance templates for visual pigments of $A_{1}$ chromophore constructed based on the cone pigments typically found in adult M. zebra: a single cone-occupying pigment (SWS1, $\lambda_{\max }=368 \mathrm{~nm}$ ) and two double cones-occupying pigments (Rh2b, $\lambda_{\max }=484 \mathrm{~nm}$; Rh2a, $\lambda_{\max }=523 \mathrm{~nm}$ ). For graphical presentation only, each of the spectra presented in (A-F) was normalized by its norm.

\section{Competing interests}

The authors declare that they have no competing interests.

\section{Authors' contributions}

SS conceived and designed the study, analyzed the data, and wrote the manuscript; SS and CWH performed all the experiments. Both authors have read and approved the final manuscript.

\section{Acknowledgements}

We thank James Mcllwain, David Berson and Daryl Parkyn for comments on the manuscript, and Suzanne Gray for assistance with underwater light flicker measurements. This research was supported by a Natural Sciences and Engineering Research Council of Canada (NSERC) Discovery Grant (10610207), NSERC Research Tools and Instrumentation Grant (359714-2008), Canada Foundation for Innovation, Ontario Innovation Trust (202821), and the Canada Research Chair Program to CWH. SS was supported by a Vanier Canada Graduate Scholarship from NSERC.

\section{Author details}

'Department of Biology, Queen's University, Kingston, Ontario K7L 3N6, Canada. ${ }^{2}$ Centre for Neuroscience Studies, Queen's University, Kingston, Ontario K7L 3N6, Canada.

Received: 10 June 2013 Accepted: 27 June 2013

Published: 4 July 2013

\section{References}

1. Bowmaker JK: Evolution of vertebrate visual pigments. Vision Res 2008, 48:2022-2041.

2. Hou XG, Aldridge RJ, Siveter DJ, Feng XH: New evidence on the anatomy and phylogeny of the earliest vertebrates. P Roy Soc B-Biol Sci 2002, 269:1865-1869.

3. Shu DG, Morris SC, Han J, Zhang ZF, Yasui K, Janvier P, Chen L, Zhang XL, Liu JN, Li Y, Liu HQ: Head and backbone of the Early Cambrian vertebrate Haikouichthys. Nature 2003, 421:526-529.

4. Collin SP, Hart NS, Shand J, Potter IC: Morphology and spectral absorption characteristics of retinal photoreceptors in the southern hemisphere lamprey (Geotria australis). Vis Neurosci 2003, 20:119-130. 
5. Collin SP, Trezise AEO: The origins of colour vision in vertebrates. Clin Exp Optom 2004, 87:217-223.

6. Collin SP, Davies WL, Hart NS, Hunt DM: The evolution of early vertebrate photoreceptors. Philos Trans R Soc Lond B 2009, 364:2925-2940.

7. Hart NS, Lisney TJ, Marshall NJ, Collin SP: Multiple cone visual pigments and the potential for trichromatic colour vision in two species of elasmobranch. J Exp Biol 2004, 207:4587-4594.

8. Theiss SM, Lisney TJ, Collin SP, Hart NS: Colour vision and visual ecology of the blue-spotted maskray, Dasyatis kuhlii Muller \& Henle, 1814. J Comp Physiol A 2007, 193:67-79.

9. Davies WL, Carvalho LS, Tay B-H, Brenner S, Hunt DM, Venkatesh B: Into the blue: gene duplication and loss underlie color vision adaptations in a deep-sea chimaera, the elephant shark Callorhinchus milii. Genome Res 2009, 19:415-426.

10. Yokoyama S, Yokoyama R: Adaptive evolution of photoreceptors and visual pigments in vertebrates. Annu Rev Ecol Syst 1996, 27:543-567.

11. Walker KR, Laporte LF: Congruent fossil communities from Ordovician and Devonian carbonates of New York. J Paleontol 1970, 44:928-944.

12. Chang BS, Crandall KA, Carulli JP, Hartl DL: Opsin phylogeny and evolution: a model for blue shifts in wavelength regulation. Mol Phylogen Evol 1995, 4:31-43.

13. McFarland WN, Munz FW: Evolution of photopic visual pigments in fishes III. Vision Res 1975, 15:1071-1080.

14. Munz FW, McFarland WN: Evolutionary adaptations of fishes to the photic environment. In Handbook of Sensory Physiology. Volume VII/5. Edited by Crescitelli F. Berlin, Germany: Springer-Verlag; 1977:193-274.

15. Neumeyer C: Evolution of color vision. In Vision and Visual Dysfunction. Edited by Cronly-Dillon JR, Gregory RL. London, UK: Macmillan, Houndsmills; 1991:282-305.

16. Osorio D, Vorobyev M: A review of the evolution of animal colour vision and visual communication signals. Vision Res 2008, 48:2042-2051.

17. Sabbah S, Troje NF, Gray SM, Hawryshyn CW: High complexity of aquatic irradiance may have driven the evolution of four-dimensional colour vision in shallow-water fish. J Exp Biol 2013, 216:1670-1682.

18. Spady TC, Parry JWL, Robinson PR, Hunt DM, Bowmaker JK, Carleton KL: Evolution of the cichlid visual palette through ontogenetic subfunctionalization of the opsin gene arrays. Mol Biol Evol 2006, 23:1538-1547

19. Ward MN, Churcher AM, Dick KJ, Laver CRJ, Owens GL, Polack MD, Ward PR, Breden F, Taylor JS: The molecular basis of color vision in colorful fish: four long wave-sensitive (LWS) opsins in guppies (Poecilia reticulata) are defined by amino acid substitutions at key functional sites. BMC Evol Biol 2008, 8:210.

20. Yokoyama S: Molecular evolution of vertebrate visual pigments. Prog Retin Eye Res 2000, 19:385-419.

21. Maximov W: Environmental factors which may have led to the appearance of colour vision. Philos Trans R Soc Lond B 2000, 355:1239-1242.

22. Darecki M, Stramski D, Sokolski M: Measurements of high-frequency light fluctuations induced by sea surface waves with an underwater porcupine radiometer system. J Geophys Res 2011, 116:C00h09.

23. Schenck H: On the focusing of sunlight by ocean waves. J Opt Soc Am 1957, 47:653-657.

24. Snyder RL, Dera J: Wave-induced light-field fluctuations in the sea. J Opt Soc Am 1970, 6:1072-1079.

25. Stramski D, Dera J: On the mechanism for producing flashing light under a wind-disturbed water surface. Oceanologia 1988, 25:5-21.

26. Kamermans M, Spekreijse H: Spectral behavior of cone-driven horizontal cells in teleost retina. Prog Retin Eye Res 1995, 14:313-360.

27. Svaetichin G, MacNichol EF: Retinal mechanisms for chromatic and achromatic vision. Ann N Y Acad Sci 1958, 74:385-404.

28. Kaneko A, Tachibana M: Retinal bipolar cells with double colouropponent receptive fields. Nature 1981, 293:220-222.

29. Kaneko A, Tachibana M: Double color-opponent receptive fields of carp bipolar cells. Vision Res 1983, 23:381-388.

30. Daw NW: Colour-coded ganglion cells in the goldfish retina: extension of their receptive fields by means of new stimuli. J Physiol (Lond) 1968, 197:567-592.

31. Fritzsch B, Collin SP: Dendritic distribution of two populations of ganglion-cells and retinopetal fibers in the retina of the silver lamprey (Ichthyomyzon unicuspis). Vis Neurosci 1990, 4:533-545.
32. Reichenbach A, Robinson SR: Phylogenetic constraints on retinal organisation and development. Prog Retin Eye Res 1995, 15:139-171.

33. Holmberg K: The cyclostome retina. In Handbook of Sensory Physiology. Volume VII/5. Edited by Crescitelli F. Berlin, Germany: Springer-Verlag; 1977:47-66.

34. Stramska M, Dickey TD: Short-term variability of the underwater light field in the oligotrophic ocean in response to surface waves and clouds. Deep-Sea Res / 1998, 45:1393-1410.

35. Sabbah S, Gray SM, Hawryshyn CW: Radiance fluctuations induced by surface waves can enhance the appearance of underwater objects. Limnol Oceanogr 2012, 57:1025-1041.

36. Parry JWL, Carleton KL, Spady T, Carboo A, Hunt DM, Bowmaker JK: Mix and match color vision: tuning spectral sensitivity by differential opsin gene expression in Lake Malawi cichlids. Curr Biol 2005, 15:1734-1739.

37. Riggs LA, Ratliff F, Cornsweet JC, Cornsweet TN: The disappearance of steadily fixated visual test objects. J Opt Soc Am 1953, 43:495-501.

38. Kelly DH: Motion and vision. 2. Stabilized spatio-temporal threshold surface. J Opt Soc Am 1979, 69:1340-1349.

39. Loew E, McFarland WN: The underwater visual environment. In The Visual System of Fish. Edited by Douglas R, Djamgoz MBA. London, UK: Chapman \& Hall; 1990:1-43.

40. McFarland WN, Loew ER: Wave produced changes in underwater light and their relations to vision. Environ Biol Fishes 1983, 8:173-184.

41. Bilotta J, Lynd FM, Powers MK: Effects of mean luminance on goldfish temporal contrast sensitivity. Vision Res 1998, 38:55-59.

42. Kelly DH: Theory of flicker and transient responses. 1. Uniform fields. J Opt Soc Am 1971, 61:537-546.

43. Kelly DH: Diffusion model of linear flicker responses. J Opt Soc Am 1969, 59:1665-1670

44. Watson AB: Temporal sensitivity. In Handbook of Perception and Human Performance. Volume 1. Edited by Boff KR, Kaufman L, Thomas JP. New York, NY: John Wiley and Sons; 1986:1-43.

45. Roufs JAJ, Blommaert FJJ: Temporal impulse and step responses of the human eye obtained psychphysically by means of a drift-correcting pertubation technique. Vision Res 1981, 21:1203-1221.

46. de Lange $H$ : Research into the dynamic nature of the human fovea-cortex systems with intermittent and modulated light. I. Attenuation characteristics with white and colored light. J Opt Soc Am 1958, 48:777-784.

47. Kelly DH: Visual responses to time-depebdent stimuli. I. Amplitude sensitivity measurements. J Opt Soc Am 1961, 51:422-429.

48. Robson JG: Spatial and temporal contrast-sensitivity functions of visual system. J Opt Soc Am 1966, 56:1141-1142.

49. Basolo AL: Female preference predates the evolution of the sword in swordtail fish. Science 1990, 250:808-810.

50. Smith AR, van Staaden MJ, Carleton KL: An evaluation of the role of sensory drive in the evolution of lake Malawi cichlid fishes. Int J Evol Biol 2012, 2012:647420.

51. Sabbah S, Lamela Laria R, Gray SM, Hawryshyn CW: Functional diversity in the color vision of cichlid fishes. BMC Biol 2010, 8:133.

52. Carleton KL: Cichlid fish visual systems: mechanisms of spectral tuning. Integr Zool 2009, 4:75-86.

53. Carleton KL, Spady TC, Streelman JT, Kidd MR, McFarland WN, Loew ER: Visual sensitivities tuned by heterochronic shifts in opsin gene expression. BMC Biol 2008, 6:22.

54. Hofmann CM, O'Quin KE, Marshall NJ, Cronin TW, Seehausen O, Carleton KL: The eyes have it: regulatory and structural changes both underlie cichlid visual pigment diversity. PLOS Biol 2009, 7:e1000266.

55. Ribbink AJ, Marsh BA, Marsh AC, Ribbink AC, Sharp BJ: A preliminary survey of the cichlid fishes of rocky habitats in Lake Malawi. S Afr J Zool 1983, 18:149-310

56. You Y, Stramski D, Darecki M, Kattawar GW: Modeling of wave-induced irradiance fluctuations at near-surface depths in the ocean: a comparison with measurements. Appl Opt 2010, 49:1041-1053.

57. Gernez P, Antoine D: Field characterization of wave-induced underwater light field fluctuations. J Geophys Res 2009, 114:C06025.

58. Oppenheim AV, Schafer RW: Discrete-time Signal Processing. 2nd edition. Upper Saddle River, NJ: Prentice-Hall; 1999.

59. Kelber A, Vorobyev M, Osorio D: Animal colour vision - behavioural tests and physiological concepts. Biol Rev Camb Philos Soc 2003, 78:81-118.

60. Cummings ME: Modelling divergence in luminance and chromatic detection performance across measured divergence in surfperch (Embiotocidae) habitats. Vision Res 2004, 44:1127-1145. 
61. Govardovskii VI, Fyhrquist N, Reuter T, Kuzmin DG, Donner K: In search of the visual pigment template. Vis Neurosci 2000, 17:509-528.

62. Dalton BE, Cronin TW, Marshall NJ, Carleton KL: The fish eye view: are cichlids conspicuous? J Exp Biol 2010, 213:2243-2255.

63. Losey GS, McFarland WN, Loew ER, Zamzow JP, Nelson PA, Marshall NJ: Visual biology of Hawaiian coral reef fishes, I. Ocular transmission and visual pigments. Copeia 2003, 2003:433-454.

64. Braekevelt CR, Smith SA, Smith BJ: Photoreceptor fine structure in Oreochromis niloticus L. (Cichlidae; Teleostei) in light- and darkadaptation. Anat Rec 1998, 252:453-461.

65. Carleton KL, Harosi FI, Kocher TD: Visual pigments of African cichlid fishes: evidence for ultraviolet vision from microspectrophotometry and DNA sequences. Vision Res 2000, 40:879-890.

66. Fernald RD, Liebman PA: Visual receptor pigments in the African cichlid fish, Haplochromis burtoni. Vision Res 1980, 20:857-864.

67. Jolly DW, Bucke D, Mawdesle LE: Anesthesia of fish. Vet Rec 1972, 91:424-426.

68. Sabbah S, Gray SM, Boss ES, Fraser JM, Zatha R, Hawryshyn CW: The underwater photic environment of Cape Maclear, Lake Malawi: comparison between rock- and sand-bottom habitats and implications for cichlid fish vision. J Exp Biol 2011, 214:487-500.

69. Lisney TJ, Studd E, Hawryshyn CW: Electrophysiological assessment of spectral sensitivity in adult Nile tilapia Oreochromis niloticus: evidence for violet sensitivity. J Exp Biol 2010, 213:1453-1463.

70. Sabbah S, Hui J, Hauser FE, Nelson WA, Hawryshyn CW: Ontogeny in the visual system of Nile tilapia. J Exp Biol 2012, 215:2684-2695.

71. Edgington ES: Randomization Tests. New York: Marcel-Dekker; 1995.

doi:10.1186/1741-7007-11-77

Cite this article as: Sabbah and Hawryshyn: What has driven the evolution of multiple cone classes in visual systems: object contrast enhancement or light flicker elimination? BMC Biology 2013 11:77.

\section{Submit your next manuscript to BioMed Central and take full advantage of:}

- Convenient online submission

- Thorough peer review

- No space constraints or color figure charges

- Immediate publication on acceptance

- Inclusion in PubMed, CAS, Scopus and Google Scholar

- Research which is freely available for redistribution 